\title{
Lack of Universality of a Psychological Problem: Epistemological Implications on Psychological Problems
}

\author{
Miika Vähämaa ${ }^{1}$ \\ Received: 17 September 2018 / Revised: 25 October 2018 / Accepted: 29 October 2018 / \\ Published online: 12 November 2018 \\ (C) The Author(s) 2018
}

\begin{abstract}
The article claims that psychological problems cannot be defined by universally meaningful psychological or philosophical scientific terminology, which leads one to consider the actual reality of psychological problems from the universal epistemological viewpoint of ordinary people. In other words, can we as laypeople or professionals reasonably claim our reality and justifiable epistemic beliefs based on the notion of a psychological problem? If psychological problems cannot be universally real beyond an idiosyncratic sense, this has important implications on how to reflect upon resolving them. Universally grounded epistemological solutions provide a sound basis to reflect upon resolving psychological problems without formal training, and the unary notion of psychological problem as such, without universal descriptive intentions, is considered with regard to (a) its common sense linguistic value as a protoepistemic concept in the context of psychologic as a theory and (b) its promising psychological and philosophical Gegenstand functional value as a notion to reflect against.
\end{abstract}

Keywords Psychological problems · Psychological language · Common sense · Psychologic · Gegenstand · Universality

\section{Introduction}

I take it as a premise that psychological problems with all their nuances are difficult or impossible to define or categorize universally in a nomothetic manner (Smedslund 1988, p. 5). Yet, such problems do exist not as matters of nomothetic essences but as matters of psychological common sense embedded in our everyday language. Therefore, psychological problems can and should be studied, explicated and - potentially-resolved through a nonempirical analysis. In order achieve the previous, we have to reconsider or at least theoretically

Miika Vähämaa

miika.vahamaa@gmail.com

1 Politics, Media and Communication Faculty of Social Science, Open University, University of Helsinki, P.O. Box 53, FI-00014 Helsinki, Finland 
reflect upon the role of psychological problems as a landmark notion in psychology. The term "psychological problems" makes sense to people in both professional and quotidian contexts. The concept is consistently utilized in multiple settings in a common sense manner, yielding understanding and some sense of universality.

The premise of this paper is the paradox that despite its frequent use, there is no universal agreement among scholars about what "psychological problem" actually means. Rather, the term is defined in multiple ways, from diagnostic manuals to philosophical discussions of normalcy, existence, and relevance of psychological problems or disorders in general. This is shown in various studies, including the debates on the American Psychological Association's Diagnostic and Statistical Manual of Mental Disorders (DSM-5) and naturalism, debates on the medicalization of phenomenological experiences, psychologization, and diagnoses and their consequences, and so on (e.g., Aftab 2016; Bolton 2008; Brinkmann 2016; Hacking 1996; Stam 2000, 2015). Because scholars radically differ in their definitions, why is there such asymmetry between the ease and fluidity that we observe in everyday life and clinical settings when we talk about "psychological problems" in such an unscientific or a proto-epistemic manner?

My answer is that while the concept seemingly escapes scientifically universal psychological or philosophical meaning or agreement (Aftab 2016, p. 11; Sass et al. 2000), one should critically consider and question the actual reality of psychological problems as such from a common sense epistemological viewpoint. By common sense epistemology, I simply mean an overarching ability to claim something as real in terms of our common culture as a justifiable shared (true) belief. Here, I side with Jan Smedslund (1988, p. 5) who postulates that from an epistemological viewpoint, common sense is the set of all implications taken for granted by all members of any given culture. It is crucial to note that such conceptualization of common sense refers to an agreement that is normally only tacit. In the domain of psychological language, the laypeople and the professionals alike are not aware of what they take for granted as they speak. It is my view, then, that such common sense epistemology applies also in the case when people speak of psychological problems.

Following Smedlund's $(1988,2012)$ thought, "proto-epistemic" here indicates merely a distinction between the everyday use of language (epistemic nature of language not yet explicated) and purposefully explicated common sense psychological language (common sense epistemic nature of language explicated) as a source of psychological knowledge. A proto-epistemic idea is a common sense idea that makes readily sense to the individual with the psychological problem and seems to be communicable to others, thus having some epistemic value as an understandable use of language, but only as common sense. Once the common sense psychological language (or some part of it) is explicated and/or formalized in a systematic manner, as in this paper, one moves from tacit knowledge of everyday psychological language to a specialized language.

This type of explicative/formalizing non-empirical research of psychological language then moves from proto-epistemic value of psychological knowledge embedded in everyday language towards an increased common sense epistemological value of knowledge attained through explicative theoretical research. From this follows Jan Smedlund's (1988, p. 5) idea, "that scientific psychological language must be identical to the language of the persons studied." This is not to undermine the scientific value of non-empirical or empirical cultural psychology, but rather to assert that psychological knowledge is readily available for all in the domain of language - the theoretical task for the cultural psychological research is to identify troublesome or intriguing issues embedded in our shared language and explicate/formalize an account to make such issues visible. 
In such a common sense manner, as argued, the concept of psychological problem seems to make sense even without being given a clear definition, preface, or reflection. Consider the common sense wisdom of Arpad Szakolczai (2013): If all suffering is pathology, but suffering is necessary for growth, then all growth becomes a problem, and all have "psychological problems." For the layperson, I contend, a psychological problem (when used as a concept of self-reflection) mostly begins as a hazy idea or feeling that something is wrong in the forementioned proto-epistemic manner.

Yet when we diagnose and categorize, we have already taken fundamental epistemological steps away from the initial problem and are perhaps well on our way to resolving it or have already done so. However, although it sounds easy, to explicate and to make visible the real nature of the psychological problems is a laborsome task and oftentimes requires the help of a therapist, a friend, or any other form of safe environment in which the problem may become to be seen in a different light, or, in other words, as a non-real entity which by necessity after such realization loses its problematic nature - the problem becomes alleviated or even resolved.

The approach taken here attempts to clarify why psychological problems are hard to define and what we may learn once we view psychological problems as matters of language use rather than essences in themselves. Also, the aim is to consider how we can serve and help the layperson with the universally applicable practices of reflection and safe interaction - both of which can be conducive to the resolution of a psychological problem.

\section{The Benefit of Seeing Psychological Problems as Non-Real}

While it may seem radical to claim or reason that psychological problems may be non-real, it is necessary in seeking not a definition, but the epistemologically universal function of psychological problem as a matter of everyday language. Such a function could explain the observable ease and fluidity in the casual use and common sense of this concept across contexts and cultures. I proceed to show that as the psychological problem concept is considered both from its common sense functionality and perhaps absurdly from its non-real nature, we can find some epistemologically sound universality in its function. The purpose of this argument is to propose a view from which we can, in a theoretically sound manner, accept both the vague and (perhaps) non-real nature of psychological problems and reflect upon how they function and facilitate our resolution of them. Can we see one of psychology's core notions, a psychological problem, as a universally non-real but nevertheless useful source of reflection from a metatheoretical viewpoint that would grant it some universal epistemological value?

To pursue an epistemologically sound and universally relevant concept transformation, I briefly discuss a few differing scholarly stances that define psychological problems. Then I consider the common sense meaning of this concept in more detail, followed by a discussion of the potential non-real nature of psychological problems lacking epistemological universality.

In the search for universal epistemological grounding in psychological problems as a functional source of reflection, I first rely on a logical and axiomatic approach to see if a psychological problem can be seen as a unary notion. I follow Jan Smedslund's (1988) psychologic and its idea that words are based on common sense, which can be a universally meaningful basis for knowledge. Words must have some invariant components - some core meaning - to matter in language and beyond contexts. The ease of explaining a psychological problem in common language can mean that a psychological problem may be impossible to define universally in scientific terminology. Due to this paradox, this common sense approach based on logical formalizations faces some contemporary challenges, as we see in due course. 
Secondly, as "psychological problems" escape universal definition, we are struck by their puzzling non-real nature as a unary notion. I reflect this nature with the concept of Gegenstand, an object that stands against the reflecting individual. Derived from German philosophy and reintroduced to psychologists by Jaan Valsiner (2012a, 2012b, Valsiner and Diriwächter 2008), the approach seems promising in terms of finding some degree of metatheoretical and epistemological universality in the notion's function. Gegenstand seems relevant and applicable as (a) it requires intentionality by the thinking agent despite the context and (b) the reflections of such problems have an effect, regardless of its particularities, on the thinking agent (Valsiner 2012a, 2012b; Winther-Lindqvist 2017, p. 165). Intentionally reconsidering the initial problem and its reality step by step and seeing it as a Gegenstand brings a sense of relief that "I may not be insane after all," or "what a good thing to consider-whether there really is any basis in reality for these peculiar worries."

The intentionality and effect of viewing a psychological problem as a Gegenstand are then discussed as the basis of reflecting psychological problems in terms of their functional validity, which can grant a universal epistemic validity from a theoretical viewpoint. Intentionality and the effect on the thinker, I propose, appear as universal, epistemologically real, and not merely phenomenological properties, which the concept of psychological problem can have. ${ }^{1}$

\section{“Psychological Problem" in Focus: Why Not Mental Disorder?}

In general terms, the question of what a psychological problem is is taken here as a matter of psychological language chosen. In other words, one may include or exclude different phenomena that either signify a psychological problem or not - depending on the theoretical/ professional terminology used. For instance, risk behaviors, social deviance, stigmatization, and the like can be viewed in some terminology as psychological problems while in some other professional terminologies, they would not count as psychological problems. In order to proceed to analyze the potential non-real nature of psychological problems - in a manner that is linguistic rather than essentialist - I conclude that a psychological problem - as an umbrella notion for a variety of psychologically problematic phenomena - is understandable through common sense. The particularities of each problem must be to some extent contextual and understandable through empirical analysis that is not the topic of this paper-whose focus is conceptual analysis. Thus, I proceed with my conceptual analysis with the following scenario with no attempt to ignore multiplicity of phenomena that may be called "psychological problems."2

When an individual comes to believe that they have a psychological problem, what do they say to themselves and others? They might use a mechanical metaphor, saying that "their mind is not working correctly," or they might refer to their biology, like "they are sick in the head" or

\footnotetext{
${ }^{1}$ Importantly, the focus here is not on the discussion of normalcy in general or in a Foucauldian sense (e.g., Rabinow 1984) or a phenomenological attempt to locate subjective meaning of what a psychological problem based on extant literature can or could mean. Rather, I take it as the result of Louis A. Sass et al. (2000), who found that psychological problems as a notion cannot attain universality in the terminological sense. Sass et al. (2000) themselves discuss features of psychopathology with regard to particulars and specific contexts, such as autism, schizophrenia, and dissociative identity disorder.

${ }^{2}$ What the exact nature of any particular psychological problem for any particular individual in any given particular context is is a matter of empirical research which is not the topic of this paper; the study at hand is concerned with non-empirical analysis.
} 
are "suffering a mental illness" or "would like to get well." They might be referred to a clinician by a legal authority due to concern by a duly authorized individual or agency, in which case a more-or-less appropriate clinical diagnosis will have been formed, e.g., agoraphobia, depression, or anxiety disorders.

Now, why do I call the above examples psychological problems rather than mental disorders, the term used in most psychiatric manuals? Perusal of this terminology in medical (mostly psychiatric, but not limited to it) and psychological journals reveals that the notions of mental disorders and psychological problems tend to be synonymous.

Yet, the further one moves away from psychiatry, the issues related to psychological difficulties experienced by an individual are more frequently described as psychological problems rather than mental disorders. For instance, gerontologists Knight and Lee (2007) define psychological problems as phobias, depression, and anxiety disorders, to mention a few examples. In their scholarly papers, dentists, interestingly, use the notion of psychological problems to cover, inter alia, the underlying causes of dry mouth and the effect of psychoactive drugs on dental health (Parks and Marek 2007). It is fairly obvious, perhaps even petty that not all professionals use these terms interchangeably; they are still highly trained healthcare professionals whose definitions of psychological problems most likely differ from the common sense meanings of laypeople. Furthermore, to the potential dismay of a critical scholar whose goal is to find a universal definition, the results would vary depending on the linguistic region and cultural system of the interpreter (e.g., Wagner, Duveen, Themel, and Verma 1999).

In my view, as vague and difficult as it is to define, the concept of psychological problem is a less technical and more common sense one than that of mental disorders, which is a more diagnostic term and hence less suitable for my generic conceptual analysis. An analogy for this choice could be to choose between "problems of the heart" and "cardiological problems." Both phrases are used by laypeople and professionals alike, even in the same contexts, but it is clear which is more commonsensical.

As I queried how psychological problems should be defined, my clinical psychologist colleagues all suggested to just see how the most recent Diagnostic and Statistical Manual of Mental Disorder (DSM-5) defines "mental disorder." Due to its high clinical and international relevance, it is purposeful to reprint the manual's definition here (italics added):

A mental disorder is a syndrome characterized by a clinically significant disturbance in an individual's cognition, emotion regulation, or behavior that reflects a dysfunction in the psychological, biological, or developmental processes underlying mental functioning. Mental disorders are usually associated with significant distress in social, occupational, or other important activities. An expectable or culturally approved response to a common stressor or loss, such as the death of a loved one, is not a mental disorder. Socially deviant behavior (e.g., political, religious, or sexual) and conflicts that are primarily between the individual and society are not mental disorders unless the deviance or conflict results from a dysfunction in the individual, as described above.

As implied, boundaries of mental disorders as defined by the DSM-5 are subject to human interests, given the criterion of "clinical significance," making it difficult for them to correspond to the natural world (APA 2013). Clinical significance is a universal characteristic of mental disorders in the DSM-5; however, it is not explicitly defined although the manual encourages the use of the World Health Organization Disability Assessment Schedule and information from family members and third parties to evaluate the clinical significance (APA 2013). Bolton (2008) explains that a study of the literature surrounding the DSM-5's 
development reveals that it refers to the idea that the condition's type and severity are such that these problems are brought to psychiatric attention (Spitzer and Williams 1982; Klein 1978). In any event, the DSM-5 description is certainly not a common sense one, but it aims at epistemological standards acceptable in the medical community at large.

The DSM-5 descriptions apparently deviate from a quotidian "layperson" perspective. For instance, the view that human biochemistry enables mental and emotional functioning is an educated one (Bolton 2008). From such a view, higher-order social functioning could be restored with the aid of medications that facilitate biochemical functions, with/or without rehabilitating therapy.

Following the line of thought put forward by Smedslund's (1988) psychological theory, we can see by examining the fields of psychology and psychiatry that attempts are made at a venerable and epistemologically sound scientific, hence universal, definition of a psychological problem. These attempts, beyond their scientific nature, have something in common: They all appear to focus on a general effort to comprehend problems via language due to the crude fact that they are written descriptions. Thus, psychological problems using advanced epistemological criteria are generally presented with this underlying notion of linguistic ability and linguistic agency for those who have and experience such problems, implying a particular nonuniversal agency (with intentionality) that is attributed to someone experiencing a "bad," "harmful," or "distressing" state, and so on (Smedslund 1981, p. 67). This must be the case if take it as true or plausible what Smedslund $(1988$, p. 6) argues regarding the social organization of psychological language ${ }^{3}$ :

The culturally created order is taken for granted unreflectively by every speaker of the given language and member of the given culture and, hence, only needs to be explicated.

This "taking for granted" can be seen in philosophically oriented theoretical psychology literature (e.g., Gallagher and Shear 1999; Neisser and Jopling 1997; Sass et al. 2000) and in more clinically oriented literature, which we have mentioned (Bolton 2008; Spitzer and Williams 1982; Klein 1978).

As expressed earlier, there is a large body of literature that discusses the nature of mental illness, psychological problems, and disorders as particular epistemological discourses, including the debates on medicalization and diagnosis and its consequences.

As we seek to explicate the proto-epistemic common sense universality behind the notion of psychological problems and proceed to think about how such rudimentary knowledge could be used in reflecting and even solving psychological problems, this paper limits its scope regarding related discourses by concluding that linguistic ability and agency are wellsupported ideas across psychiatry and psychology.

Therefore, for the purposes of this paper, I will make use of one ambitious attempt by Bolton (2008, p. xiii; italics added) to describe a psychological problem:

Typically the experiences of the people with problems are of anguish, confusion, of being in some respects and to some degree unable to carry on with their normal lives. In other kinds of cases, the problem is less for the persons themselves and more for others, in the form of threats to safety from behavior that deviates from fundamental standards of conduct. These are the kinds of phenomena that trigger involvement of mental health services and diagnosis of mental disorder.

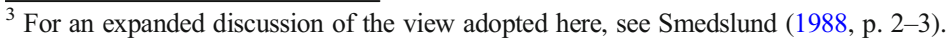


In what follows, I first consider psychological problems from the viewpoint of a layperson with "problems" that hinders her from carrying out her "normal life" (Bolton 2008). I examine this proto-epistemic (not yet explicated) view of a psychological problem through the lens of common sense psychologic and discuss the value of recognizing what is common for everyone.

\section{Common Sense of Psychological Problems: Potentially Common Help?}

As noted, we have no sound basis to pursue a universal scientific definition for a psychological problem as a unary term. Rather, it suffices to say that one important feature should be drawn from the depicted nomenclature (in addition to their reliance on language) surrounding "psychological problems": they typically mark either the relation to the particular agent (an individual with issues) or to the particular respects of the problem (Bolton 2008, p. xiii). Hence, as most of the literature shows, we can theoretically and empirically argue that psychological problems exist in relation to some specific individuals with some issues that culture regards as "bad" or "harmful," warranting an appropriate intervention, help, or even diagnosis.

Without the unary scientific existence or agreed-upon reality of a psychological problem, we are stuck with an epistemological dilemma: On what grounds can we metatheoretically reflect upon psychological problems? Based on Smedslund's (1988, 2012) psychologic, the unary notion of a psychological problem should have a universally sound epistemic basis in its common sense linguistic value. In short, psychological problems make sense to people without professional training: It has been subsumed in our common knowledge and vocabulary. ${ }^{4}$

Given the proto-epistemic common sense universality of these problems, can we somehow reflect upon a resolution of such problems outside of a particular fine-tuned and specialized epistemological discourse in order to provide some common sense help and tools of reflection for anyone?

\section{The Premises of Psychologic}

Psychologic as a non-empirical approach to psychological language presumes that we as cultural psychologists, thinkers, and laypeople continue to express invariant elements of word meanings in our contemporary world (Smedslund 1988, 2012). Theoretically, words have stagnant, permanent meanings for all competent users of a language. Some such elements in psychologic are the "semantic primes"- the conceptual basis of language and therefore of psychologic (for a detailed discussion on semantic primes, see Smedslund 2012). As Smedslund (2008, p. 160) summarizes:

There must be invariant components in word meaning in order to explain the usefulness of languages and their function in social life. If words were completely transparent, that is, their meanings completely determined by context, the orderliness of social life could not be explained. Part of the function of language is precisely to ensure communication with little contextual support.

\footnotetext{
$\overline{{ }^{4} \text { Here, one must again recognize }}$ the cultural differences. Our focus here is on English language, and our common-sense vocabulary may differ from other languages, as Wagner (1999) and colleagues posit.
} 
While this statement clearly outlines the necessity of constants in word meanings, some suspicion arises as to whether word meanings "must be" invariant. The immediate challenge of common sense epistemological universality in our word meanings in general, and in psychological problems in particular, comes from the recent developments of our mass and social media practices. I will briefly defend the view of stability of psychological language by considering the challenges of shared language (particularly word meanings) that remains permanent and to some extent invariable come from changes in media practices.

Instability of language occurs, according to some authors, in how we use language and word meanings in an increasingly idiosyncratic manner (Oeberst et al. 2016, pp. 105-106). The arguments is that in a world where Web 2.0 applications have become a primary source of news (Gottfried and Shearer 2016), and the primary social media app Facebook allows your friends to influence your newsfeed (Baresch et al. 2011, p. 18; Flaherty 2011, pp. 1302-1303). From this view, language is often transitional and transformational as times and social practices change (e.g., Beckstead 2010; Greco and Stenner 2017; Salvatore and Venuleo 2017; Stenner, Greco, and F. Motzkau 2017).

Are there words, then, that all agents skilled in a language know ab initio, or are we entering a different type of semantic reality altogether (Baudrillard 1988, p. 145)? To answer this question, it can be reasonably claimed that common word meanings that form linguistic "common sense" are the sum of multiple higher cultural psychological and societal functions. Word meanings are an attempt to make something commonly understandable: language, particularly as we discuss psychological problems, is certainly intentional and goal-oriented and therefore relatively stable. As Smedslund (1988, p. 104; 2012) postulates with regard to the stability of language and the psychologic dependent upon such stability:

It is well known that cultures and their languages change over time. ... There are two reasons for believing that the present system must be relatively stable. The first is that societal changes must always involve considerable translatability in order to preserve the conditions for orderly interaction and communication. The second is that the concepts described in the present system appear to be of such a fundamental nature that it is hard to see how they could change very much.

Most concretely, we can observe the continuing role of common sense in everyday social interactions. Even in small group settings, we intend to be logical in a coherent sense with our understanding of the world and how we imagine that it exists for us within our momentary conceptual frameworks (Smedslund 2012, p. 295).

All things considered, the good news is that we see that communication can be functional only because of common word meanings that make up the "common sense" even if only to a limited extent. As Habermas (1994, p. 116) and Smedslund (1988) argue, holding a shared language leads with its constraints individuals to develop a common sense of meaning in the groups within which they communicate. Via this sensus communis, individuals can still operate with ease and fluidity in the symbolic realm (Smedslund 2012).

\section{Psychological Problems, Proto-Epistemology, and Psychologic}

While it is true that language is constant enough for continuous common sense, I conclude that it is not possible to both scientifically and commonsensically define the terms "psychological" and "problem," whether they appear separately or together. 
By the strict epistemological standards of psychological theory, the notion of a psychological problem is too elliptical, ephemeral, and idiosyncratic in meaning in order to give it a scientific definition. Meanings, as we know from everyday experience, are often circulated in small-group interpersonal communication with particular customs and conventions.

Yet, there is this simple idea of a core meaning attached to every word, as Smedslund (2012) argues, and therefore we understand why our term is still so easily understood as a notion of common sense. We as scholars must rely on common sense, too.

The readers of this text will remember the always-extant corpus of word meanings that was previously discussed. When that corpus of signifiers disintegrates, the semiotic realm itself will dissipate; we actually cannot imagine the consequences of this since imagination itself exists in the semantic realm and is "a fundamental higher psychological function that is devoted to the manipulation of complex wholes of iconic and linguistic signs" (Tateo 2015, p. 146; see also Brinkmann 2015; Harris 2000). Hence, the potentially universal value in the phrase "psychological problem" will most likely keep escaping more formal universal epistemological criteria.

While some word meanings remain invariant, this takes place more than before in idiosyncratic or specialized settings, as in this carefully curated article. Simultaneously, invariant components of word meanings have become decreasingly universal in the generally common, public sphere and non-particular settings (Habermas 1984, 1994). How, then, could we make use of the psychological problem and imagine how laypeople and trained psychologists could use such basic psychological language for self-reflection and potentially for solving psychological problems?

Importantly, we rely on imagination to seek some universally sound epistemological value beyond the proto-epistemic for psychological problems. Imaginative processes may yield some universal base as a source of epistemological validation. Imaginary processes such as reflection deal even with non-real entities. This is relevant since our initial puzzle was to consider how we could credibly view psychological problems as non-real with the lack of universal scientific epistemic standards to define them. In some critical sense, therefore, psychological problems are rendered non-real.

In what follows, the philosophical concept of Gegenstand is discussed as a way to find some epistemological universality in the notion of a psychological problem as a source of theoretical and quotidian reflection.

\section{Psychological Problem Reflected as Gegenstand}

Common sense, with its related notion that some things can be universally understood due to their proto-epistemic value, remains important even if it is only something to reflect against: to evaluate our habits of reasoning, to reflect the regulation of our practices (Meinong 1960, 1983). In such a way, common sense and any familiar but abstract concepts to that end remain as objects of the reflective imaginative process. A Gegenstand is a counterforce to our intentions and thoughts that enables and keeps us thinking when the word meanings change.

Stemming from German philosophy and early psychology, Gegenstand has been reintroduced to psychologists as a metatheoretical global idea. Valsiner and Diriwächter's definition would not allow the differentiation of "a psychological problem" from any other trigger-for-meaning key concept (Valsiner 2012a, 2012b; Valsiner and Diriwächter 2008). ${ }^{5}$

\footnotetext{
${ }^{5}$ While my aim is not the exegesis of the early (or later) psychological accounts of Gegenstand, it should be noted that the meaning of Gegenstand appears in the earlier literature as any possible "irritation" in the psychological life of individual.
} 
What, then, is the specificity or promoted theoretical and epistemological value of a psychological problem over any other meaning-unit, one might wonder? My answer: A psychological problem, as we have considered, can be commonly understood, is a frequently used notion of psychology and could be woefully useful concept for reflection. At least, it seems to me, psychological problems are arguably more relevant "triggers" than, say, dropping your wallet, which in a more global reading of Gegenstand is an equally relevant psychological event as any other.

I will consider the role of Gegenstand with a more precise example, but the focus remains clear: The topic of this analysis is not to define a psychological problem, but to examine its common sense reality/non-reality and determine how Gegenstand could be useful in reflecting upon psychological problems if we consider psychological language to be a relevant topic in cultural psychology. ${ }^{6}$

As Valsiner (2014, p. 153) and Winther-Lindqvist (2016, 2017) describe the phenomenon Gegenstand: lost or non-real words keep carrying significance, their meanings can remain, if only to make us re-evaluate and provide Gegenstand to "stand against us" as a thought in a philosophical and psychologically reflective sense. Building on Valsiner (2014), Luca Tateo (2018, p. 5) describes such non-real notions, the Gegenstand:

Their "reality" in psychological terms is undeniable, or, as Meinong says in an elegant and famous paradox: "there are objects of which it is true that there are no such objects" (Meinong, 1960, p. 82). In other words, if one can think, feel or desire something, this "something" once invested with the intentionality of the agent becomes an "objective" (a psychological fact) that "always seems to require in turn an Object which has being" (Meinong, 1960, p. 85). The crucial role in this transformation is the role of the agent: by acting upon things in nature, these become objects.

These objects can resist our actions (stand against us $=$ Gegen + stand), or can evade us (Valsiner 2014, p. 153).

\section{Gegenstand Yields Universality}

According to Valsiner (2014), any psychological act thus generates a new totality, a Gegenstand including (a) an assumed set of properties, (b) the object to which the properties refer, and (c) the intentionality of the agent (see also Tateo 2018, p. 5). We can follow this line of reasoning and consider the psychological problem to be the result of an act, given that it is a product of our reflection and imagination. To any given individual, the particular properties parts $\mathrm{a}$ and $\mathrm{b}$ in this reasoning are idiosyncratic, while the intentionality (part [c]) of the reflecting agent is universal. Hence, we can build the argument that as a Gegenstand, a psychological problem has some universal epistemological basis as an object upon which any linguistic and reflective agent must intentionally rely.

If viewed as a Gegenstand, we accept that a psychological problem is a non-real notion in a universal terminological sense. However, a psychological problem reveals itself as relevant and real in the universal sense due to the intentionality of the reflection and imagination (which are functions) required by the linguistic agent. According to Valsiner (2014) and Tateo (2018),

\footnotetext{
${ }^{6}$ One could take an alternative view here. A potential source of criticism is my focus on the internal intentionality of the linguistic agent who may define what a psychological problem means for themselves. A critique may question whether we are not just stamping problem labels from the outside. If we adopt the less global approach to Gegenstand from a psychological point of view, we still keep the focus on seeking universally and epistemologically intriguing and sound ways to view and discuss psychological problems.
} 
this matters epistemologically: Once an individual invests in, approaches, or moves towards an object (intentionality), they become Gegenstanden and acquire some specific relational properties with respect to the agent.

Via this process, universally non-real and non-existing objects, in not only our case but in multiple others ("freedom," "nation," "Tooth Fairy," "race," etc.), attain a universal epistemological function as existing things that exert some action upon the world in a universal sense through the agent's intentional action of attaching idiosyncratic and particular meanings and feelings to these objects. In sum, Tateo (2018, p. 5; italics added) posits:

We do not just "see" a thing, we always see a thing "as" something, actively selecting some properties and neglecting others, relating to it by the light of our preferences, affects, idiosyncrasies. We take a position towards the object, we "stand against" it and in return the object becomes a Gegenstand (stands against us).

While it is easy to see that any one of us would have a particular idiosyncratic nomenclature to understand what a psychological problem means, we must also consider the implications of the universal epistemic value of intentionality that our reflection requires. As presented above, once we reflect upon a psychological problem, we use our imagination (Tateo 2017). Whatever the particularities of the problem may be, we do the reflecting intentionally, and such reflection elicits a psychological effect upon us in the form of Gegenstand. This effect seems to be a universal psychological phenomenon (Winther-Lindqvist 2017, p. 165; 2016).

\section{Psychological and Non-Psychological Problems}

When we are intentional about a psychological problem, we immediately imply the complementary linguistic array of non-psychological problems. This open-ended linguistic set of meanings is obviously idiosyncratic regarding the reflecting agent. However, the language itself constrains and confines what idea can be in commonsensical manner spoken of as a psychological problem and what idea does not make sense if referred to as a psychological problem as we speak (see Smedslund 1988, p. 6; 104-105).

If we agree that we can intentionally reflect upon psychological problems as a unary notion, we acknowledge that in an equal sense, there is a linguistic set of non-psychological problems to which we are not referring or are not intentionally reflecting upon. This realization, I contend, gives further universal credence to the idea that only through the intentional use of language and reflection can we grant a psychological problem some universal epistemological basis. $^{7}$

\section{The Psychological Effect of Gegenstand}

Some degree of epistemological universality that could stand for further scientific scrutiny of a psychological problem, then, lies in the fact that reflecting upon the problem has to be intentional regardless of the idiosyncrasies and that such reflection has a real effect on the thinking agent (Valsiner 2015; Winther-Lindqvist 2017, p. 165; 2016). This effect is not particular but universal as such. A psychological problem as a Gegenstand starts with intentional reflection, and through its

\footnotetext{
${ }^{7}$ Following this line of argumentation, we can thus posit that there are non-universal, non-psychological problems that we refer to by default, but only in the sense that we intentionally limit our focus and reflection upon A (a psychological problem) and not on the non-A (a non-psychological problem).
} 
effect, forces the thinking agent to use a set of meanings that can be shared with others. Therefore, the Gegenstand value of a psychological problem yields universal epistemic value as a function because its approach opens a general pathway to consider solving or reflecting psychological problems, not only as particular non-universal issues but with a degree of epistemological universality.

Intentional reflection is the start, but interestingly, it affects the thinking agent. In Tateo's words, reflection with regard to objects that do not have any real epistemological basis beyond their Gegenstand value is reciprocal (Tateo 2018, p. 10):

When an object (existing or non-existing, or not-yet existing, etc.) is invested by an agent directionality starts to "resist" it. Our intentionality exerts a grip on "objectives," but in return they exert a grip on us.

To exemplify this point, we can consider Sass et al.'s (2000) reflection on the reality of an experience in the context of dissociative identity disorder. While the example is radical, we can see through this scenario that what has a real psychological effect is a matter of the speaker's intentionality. The reality of a psychological object of thought is a matter of ability in using language with an intention to reflect upon a psychological object given the fact that such intention is meant to make sense in one's linguistic community. As Sass et al. (2000, p. 92; italics added) discuss in the framework of dissociative identity disorder,

...if this subject really doesn't remember, and really is psychologically discontinuous with the original subject of experience, then this subject is - precisely in virtue of these discontinuities - a different subject, even if she and the original subject share a body. So we cannot conclude from the fact that something happened to her body that her failure to acknowledge that happening amounts to false consciousness on her part.

Here, the real or non-real nature of a psychological problem is a matter of one being able to intentionally assign linguistic meaning to the notion of a psychological problem and experience its effect in terms that make sense to the thinking agent and others. In sum, to reflect upon a psychological problem as a universal notion, we have some degree of epistemological basis in the intentionality of the linguistic and reflective agent that is universally real. This intentionality entails reciprocity as the object of reflection affects the reflecting agent.

This effect can be viewed as the universal epistemological function of the object, the psychological problem. But, like the scenario depicted above, we would have to become different subjects time and again, with endless iterations, to comprehend the subjective idiosyncrasies that always accompany the notion and its effect on the one who is reflecting.

Thus, taken together, the presented Gegenstand approach of psychological reflection, in some sense, comes close to philosophical phenomenology as Meinong (1960) has posited. But it differs in its articulated idea that some significant degree of epistemological universality and non-subjective experience can be found through the intentional reflection of the object, the Gegenstand, and its reciprocal effect (Bayne and Montague 2011; Kriegel and Williford 2006; Smith and Thomasson 2005).

\section{Reflecting on Solving Psychological Problems}

How, then, is the psychological problem resolved? As suggested above, it is a commonly understood and linguistically problematic notion, a false consciousness arising around an epistemic question. We perceive objects not as things-in-themselves (ding-an-sich), but as investments 
with meaning based on prior experiences and memories. Usually, these associations enrich our lives, but they are sometimes alarming, paralyzing, or profoundly detrimental. To follow Smedslund's (1981, p. 67) argumentation, it is possible to resolve such unwanted psychological problems through intentional learning what is actually already (implicitly) known by the individual experiencing the problem - resolving the problems is, in such cases, to explicate the implicit commonsensical knowledge which may help to overcome the psychological problem by seeing it as non-real Gegenstand in the light of newly gained knowledge:

The fact that a problem remains unsolved, even though the person wants to solve it, means that the person cannot in fact manage what is required and/or does not, for some reason, try to.

Obviously, the approach taken here requires safe and productive conditions of interaction in order to create a chance for an individual to become aware of the false nature of the initial problem. A failure to create such a safe atmosphere can happen in a clinical setting as well as in any quotidian interactions. The point is that once the interaction is aiming at explicating the implicit knowledge to yield an epistemological insight of the non-real nature of the beliefs creating the problem (to project them as Gegenstand), such attempts can succeed or fail. Smedslund (1981, p. 67) posits the following regarding the failed attempts to explicate the implicitly known nature of psychological problems, as he discusses the conducive conditions for psychological learning:

This, again, must mean that the conditions for acquiring this knowledge and/or this skill cannot have been available. Since a precondition of most learning is that overt attempts can be made, there must have been a suppression of trying. One very frequent cause of such suppression is a lack of interpersonal safety (trust).

To ground our analysis with a practical example, we can consider our general self-doubt. According to Bolton, the experiences of "people with problems," quite commonly, "are of anguish, confusion, of being in some respects and some degree unable to carry on with their normal life" (Bolton 2008, p. xiii: italics added). For instance, we can posit that psychological factors lie at the heart of forming a fear of a personal appearance that is "normal." Self-doubt associated with identity conflicts and role choices is thought to lead to conflicts that are resolved through transferences, including painful somatic urges in extreme cases, as research shows (Silverstein et al. 1993, p. 652; Gordon 2000, 2001).

\section{Seeing the Initial Problem as Non-Real}

From our approach, psychological problems are resolved through a laborious process in which the individual comes to see the initial problem as non-real. The remains of the psychological problem are never fully removed or erased, but serve as a Gegenstand. To overcome a psychological problem, from this perspective, is to come to terms with the initial psychological problem's non-real nature, for which there was never any epistemological basis beyond the common sense proto-epistemic realization that "I have a problem of a psychological nature."

Our example of personal self-doubt can create unwanted, problematic consequences, i.e., being afraid of giving a public presentation. In such a scenario, the psychological problem experienced by the individual is that of anguish related to performing a public presentation. The "problem" prevents the individual from doing something they would normally do with no problem. How could one use the lessons learned from explicated common sense of such a psychological problem when seen as Gegenstand? 
The individual could intentionally reflect the nature of performing in public. As they place the initial anguish into the more elaborated and narrowed-down fear, it can become Gegenstand, projecting anguish stemming from the initial anguish. Yet, by intentionally accepting the anguish and projecting it as an idea instead of fact or reality, a pathway is opened to distance oneself from the initial anguish without denying it. The initial anguish remains as a Gegenstand, something to reflect against that will continue to have a reciprocal effect on the thinker. However, the hope in such an approach is that the understanding of the reality and necessity of the initial anguish may start to dissolve via such psychological reflection.

The metatheoretical perspective shows that as a Gegenstand, the psychological problem remains. This validates the individual to intentionally reflect a particular problem and experience its psychological effect while acknowledging, perhaps paradoxically, the non-real nature of the initial problem.

The psychological problem's lack of universality thus grants an interesting vantage point: its universal and epistemological reality in a functional sense. Some degree of epistemological universality of a psychological problem as a unary notion is therefore found with (a) the intentionality of the agent who reflects a psychological problem as an object, or Gegenstand, and (b) the necessary psychological effect that such reflection of this abstract object has on the individual.

\section{Conclusions}

To conclude, I argue that psychological problems cannot be meaningfully defined by scientifically universal psychological or philosophical terminology, which questions the actual reality of psychological problems from an overall universal epistemological viewpoint. Following Smedslund's psychologic, psychological problems cannot be epistemologically universalized by some post hoc scientific criteria. Yet, psychological problems carry out an important, a priori, epistemic function as common sense - as our everyday language that can be explicated for purpose of psychological research.

Initially, we were struck with the non-real nature of unary psychological problems, as common sense nature of psychological language leads one to question whether psychological problems are actually scientifically real at all. When viewed from another theoretical viewpoint, it was argued that as Gegenstand, an object of intentional reflection that affects the thinking agent, important epistemological universality was found in the functionality of the notion of "psychological problem." This is radical since both a professional psychologist and a layperson would render psychological problems as obvious matters, while epistemologically they are hardly definable, even rendering themselves as non-real.

The Gegenstand approach, then, allows us to reflect on the non-real nature of psychological problems - real only as common sense and undefined by a universal scientific consensus - and use this theoretical approach to explicate, to reflect, and even to solve psychological problems. It is posited that resolving a psychological problem is fundamentally a process in which one comes in terms with of the non-real epistemological nature of the initial psychological problem. The understanding of the common sense epistemological nature of psychological problems is a start: it is a way to point our attention to the problem. What remains after that is reflection, a form of psychological learning that warrants safe conditions in order to take place. The goal, it was argued, is to see the non-real epistemic nature of the initial problem and to 
grant the initial psychological problem the status of Gegenstand. The Gegenstand, it is argued, remains with the individual as an object of thought and projection while the initial psychological problem is resolved by seeing the problem's original and harmful nature as epistemologically non-real, remaining only as reflective Gegenstand.

In sum, the lack of universality of understanding what a psychological problem is can paradoxically lead us to consider the universal components carried out by the entire notion of a psychological problem. If we cannot say precisely what a psychological problem is, the idea of it can in turn become a valuable tool to guide our reflection on the nature of other, perhaps even more crucial matters. It is argued that the reflective Gegenstand value of a psychological problem can actually remain universal and epistemologically real in a functional sense. The lack of universality in our case, then, means that we can find some degree of epistemological universality from the intentionality of the agent who reflects a psychological problem as an object and from the necessary effect that such reflection of this object has on the individual.

Acknowledgements Open access funding provided by University of Helsinki including Helsinki University Central Hospital.

\section{Compliance with Ethical Standards}

Conflict of Interest The authors declare that they have no conflict of interest.

Open Access This article is distributed under the terms of the Creative Commons Attribution 4.0 International License (http://creativecommons.org/licenses/by/4.0/), which permits unrestricted use, distribution, and reproduction in any medium, provided you give appropriate credit to the original author(s) and the source, provide a link to the Creative Commons license, and indicate if changes were made.

Publisher's Note Springer Nature remains neutral with regard to jurisdictional claims in published maps and institutional affiliations.

\section{References}

Aftab, A. (2016). Mental disorders and naturalism. The American Journal of Psychiatry - Residents' Journal, $11(3), 10-12$.

American Psychiatric Association. (2013). Diagnostic and statistical manual of mental disorders (5th ed.). Washington: DC.

Baresch, B., Knight, L., Harp, D., \& Yaschur, C. (2011). Friends who choose your news: An analysis of content links on Facebook. ISOJ: The Official Research Journal of International Symposium on Online Journalism, 1(2), 1-24.

Baudrillard, J. (1988). The hyper-realism of simulation. In M. Poster (Ed.), Jean Baudrillard: Selected Writings (pp. 143-147). Stanford, California: Stanford University Press.

Beckstead, Z. (2010). Commentary: Liminality in acculturation and pilgrimage: when movement becomes meaningful. Culture \& Psychology, 16(3), 383-393.

Bayne, T., \& Montague, M. (Eds.). (2011). Cognitive phenomenology. Oxford and New York: Oxford University Press.

Bolton, D. (2008). What is mental disorder? An essay in philosophy, science, and values. Oxford: Oxford University Press.

Brinkmann, S. (2015). Imagining cultural psychology. Culture \& Psychology, 21(2), 243-250.

Brinkmann, S. (2016). Diagnostic Cultures: A Cultural Approach to the Pathologization of Modern Life. New York: Routledge. 
Flaherty, D. K. (2011). The vaccine-autism connection: a public health crisis caused by unethical medical practices and fraudulent science. Annals of Pharmacotherapy, 45(10), 1302-1304.

Gallagher, S., \& Shear, J. (Eds.). (1999). Models of the self. Thorverton: Imprint Academic.

Gordon, R. A. (2000). Eating disorders: Anatomy of a Social Epidemic (2nd ed.). Malden, MA, US: Blackwell Publishers Inc.

Gordon, R. A. (2001). Eating disorders East and West: a culture-bound syndrome unbound. In M. Nasser, M. A. Kazman, \& R. A. Gordon (Eds.), Eating disorders and cultures in transition (pp. 1-16). New York: Brunner-Routledge.

Gottfried, J., \& Shearer, E. (2016, May). News Use Across Social Media Platforms 2016 (Publication by Pew Research Center). Retrieved from http://www.journalism.org/2016/05/26/news-use-across-social-mediaplatforms-2016/

Greco, M., \& Stenner, P. (2017). From paradox to pattern shift: conceptualising liminal hotspots and their affective dynamics. Theory \& Psychology, 27(2), 147-166.

Habermas, J. (1984). The theory of communicative action. Reason and the rationalization of society. Vol 1. London: Heinemann.

Habermas, J. (1994). The past as future. Cambridge, MA: Polity Press.

Hacking, I. (1996). The Looping Effects of Human Kinds. In D. Sperber, D.Premack, \& A. J. Premack (Eds.), Causal Cognition: A Multidisciplinary Debate (pp. 351-383). Oxford: Oxford University Press.

Harris, P. L. (2000). Understanding Children's worlds. The work of the imagination. Malden: Blackwell Publishing.

Klein, D. F. (1978). A proposed definition of mental illness. In R. L. Spitzer \& D. F. Klein (Eds.), Critical Issues in Psychiatric Diagnosis (p. 41). New York: Raven Press.

Knight, B. G., \& Lee, L. (2007). Mental health. In Encyclopedia of gerontology (pp. 159-166). New York: Elsevier.

Kriegel, U., \& Williford, K. (Eds.). (2006). Self-Representational Approaches to Consciousness. Cambridge, Masssachusetts: MIT Press.

Meinong, A. (1960). The theory of objects. In R. M. Chisholm (Ed.), Realism and the background of phenomenology (pp. 76-117). New York: The Free Press of Glencoe.

Meinong, A. (1983). On assumptions. Berkeley: University of California Press.

Neisser, U., \& Jopling, D. (Eds.). (1997). The conceptual self in context: Culture, experience, self-understanding. Cambridge/New York: Cambridge University Press.

Oeberst, A., Kimmerle, J., \& Cress, U. (2016). What is knowledge? Who creates it? Who possesses it? The need for novel answers to old questions. In U. Cress, J. Moskaliuk, \& H. Jeong (Eds.), Mass collaboration and education. Computer-Supported Collaborative Learning Series, vol 16 (pp. 105-124). Cham: Springer.

Parks, E. T., \& Marek, C. (2007). Managing the patient with psychological problems. In S. J. Stefanac \& S. P. Nesbit (Eds.), Treatment planning in dentistry (pp. 367-389). New York: Elsevier.

Rabinow, P. (Ed.). (1984). The Foucault reader. New York: Pantheon.

Salvatore, S., \& Venuleo, C. (2017). Liminal transitions in a semiotic key: the mutual in-feeding between present and past. Theory \& Psychology, 27(2), 215-230.

Silverstein, B., Perlick, D., Clauson, J., \& McKoy, E. (1993). Depression combined with somatic symptomatology among adolescent females who report concerns regarding maternal achievement. Sex roles, 28(11-12), $637-653$.

Sass, L. A., Whiting, J., \& Parnas, J. (2000). Mind, self and psychopathology: reflections on philosophy, theory and the study of mental illness. Theory \& Psychology, 10(1), 87-98.

Smedslund, J. (1981). The logic of psychological treatment. Scandinavian Journal of Psychology, 22, 65-77.

Smedslund, J. (1988). Psycho-logic. Berlin: Springer-Verlag.

Smedslund, J. (2008). From Heider to psycho-logic. Social Psychology, 39(3), 157-162.

Smedslund, J. (2012). Psycho-logic: Some thoughts and after-thoughts. Scandinavian Journal of Psychology, 53(4), 295-302.

Smith, D. W., \& Thomasson, A. L. (2005). Phenomenology and philosophy of mind. Oxford and New York: Oxford University Press.

Spitzer, R. L., \& Williams, J. B. (1982). The definition and diagnosis of mental disorder. In W. R. Grove (Ed.), Deviance and mental illness (pp. 19-20). Beverly Hills: Sage.

Stam, H. J. (2000). Theorizing Health and Illness: Functionalism, Subjectivity and Reflexivity. Journal of Health Psychology, 5(3), 273-283.

Stam, H. J. (2015). The historical boundedness of psychological knowledge and the ethics of shared understandings. Journal of Theoretical and Philosophical Psychology, 35(2), 117-127.

Stenner, P., Greco, M., \& Motzkau, J. F. (2017). Introduction to the special issue on liminal hotspots. Theory \& Psychology, 27(2), 141-146. 
Szakolczai, A. (2013). The social pathologies of contemporary civilization: meaning-giving experiences and pathological expectations concerning health and suffering. In K. Keohane \& A. Petersen (Eds.), The social pathologies of contemporary civilization (pp. 25-43). London and New York: Routledge.

Tateo, L. (2015). Giambattista Vico and the psychological imagination. Culture \& Psychology, 21(2), 145-161.

Tateo, L. (2017). Seeing imagination as resistance and resistance as imagination. In N. Caudhary, P. Hviid, G. Marsico, \& J. W. Villadsen (Eds.), Resistance in everyday life: constructing cultural experiences (pp. 233246). Singapore: Springer.

Tateo, L. (2018). Affective semiosis and affective logic. New Ideas in Psychology, 48, 1-11.

Valsiner, J., \& Diriwächter, R. (2008). Conclusion: Returning to the whole: a new theoretical synthesis in the social sciences. In R. Diriwächter \& J. Valsiner (Eds.), Striving for the Whole: Creating Theoretical Syntheses (pp. 211-237). Somerset, NJ: Transaction Publishers.

Valsiner, J. (2012a). A guided science: history of psychology in the mirror of its making. New Brunswick: Transaction Publishers.

Valsiner, J. (2012b). Psychology courting culture: Future directions and their implications. In J. Valsiner (Ed.), Handbook of culture and psychology (pp. 1092-1104). Oxford: Oxford University Press.

Valsiner, J. (2014). Functional reality of the quasi-real: Gegenstandstheorie and cultural psychology today. Culture \& Psychology, 20, 285-307.

Valsiner, J. (2015). Generalization is possible only from a single case (and from a single instance). In B. Wagoner, N. Chaudhary, \& P. Hviid (Eds.), Integrating experiences: Body and mind moving between contexts (pp. 233-244). Charlotte: Information Age Publishers.

Wagner, W., Duveen, G., Themel, M., \& Verma, J. (1999). The modernization of tradition: thinking about madness in Patna, India. Culture \& Psychology, 5(4), 413-445.

Winther-Lindqvist, D. (2016). Time together-time apart: nothingness and hope in teenagers. In J. Bang \& D. Winther-Lindqvist (Eds.), Nothingness (pp. 143-167). New Brunswick: Transaction.

Winther-Lindqvist, D. (2017). Hope as fantasy: an existential phenomenology of hoping in light of parental illness. In B. Wagoner, I. Bresco, \& S. Awad (Eds.), The psychology of imagination: History, theory and new research horizons, Volume 3 of Niels Bohr Professorship Lectures series (pp. 151-173). Charlotte: Information Age Publishers. 